\title{
Promoção da saúde: discursos e concepções na atenção primária à saúde
}

\author{
Health promotion: discourses and conceptions in primary \\ health care
}

\author{
Joni Marcio de Farias ${ }^{1}$ (D), Luana Cademartori Minghelli', Jacks Soratto' ${ }^{1}$ \\ ${ }^{1}$ Universidade do Extremo Sul Catarinense (UNESC) - Criciúma (SC), Brasil.
}

\begin{abstract}
Como citar: Farias JM, Minghelli LC, Soratto J. Promoção da saúde: discursos e concepções na atenção primária à saúde. Cad Saúde Colet, 2020;28(3):381-389. https://doi.org/10.1590/1414-462X202028030351
\end{abstract}

\section{Resumo}

Introdução: Apesar de inicialmente o termo promoção da saúde ser utilizado para caracterizar um nível de atenção da medicina preventiva, ele foi se ressignificando ao longo do tempo. Objetivo: Objetivo deste estudo foi identificar o conhecimento sobre promoção da saúde dos profissionais de saúde do município de Criciúma - SC. Método: Trata-se de um estudo observacional descritivo com abordagem qualitativa, que contemplou pesquisa com 123 profissionais de saúde de 28 Estratégias Saúde da Família e 1 Núcleo Ampliado de Saúde da Família e a Secretária Municipal de Saúde. O instrumento de coleta de dados utilizado foi um questionário autoaplicável contendo sete perguntas abertas. A análise dos dados foi realizada segundo a análise temática de conteúdo e por meio de estatística descritiva e auxílio do software Atlas.ti com a inserção de documentos, trechos de falas, códigos, agrupamento por proximidade temática e redes de visualização. Resultados: Os dados geraram 137 trechos de respostas 11 códigos que foram agrupados em 4 categorias: modelo preventivo (36,6\%), modelo educativo $(29,2 \%)$, modelo positivo de saúde $(28,5 \%)$ e modelo de articulação, participação e controle social $(5,8)$. Conclusão: $O$ entendimento dos profissionais de saúde sobre a promoção da saúde está predominantemente relacionado a visão preventivista, ligada principalmente à prevenção de fatores de risco e doenças.

Palavras-chave: promoção da saúde; atenção primária à saúde; política de saúde.

\begin{abstract}
Background: Although initially the term health promotion is used to characterize a level of care of preventive medicine, it has been resignifying over time. Objective: This study aimed to identify knowledge about the health promotion of health workers in the city of Criciúma - SC. Method: This is a descriptive observational study with a qualitative approach, which included 123 professionals from 28 Family Health Strategies and 1 Family Health Support Center and at the Municipal Health Secretary. The thematic analysis of content analyzed the data, divided into three phases and using descriptive statistics, using the software Atlas.ti, with the insertion of documents, excerpts of the speech, codes, grouping by proximity thematic and visualization networks. Results: Data generated 137 quotations, 11 codes that were grouped into 4 categories: a preventive model [36,6\%], an educational model $[29,2 \%]$, a positive health model $[28,5 \%]$ and a model of articulation, participation and social control $[5,8]$. Conclusion: the understanding of health workers about health promotion is predominantly related to the preventive view, mainly related to the prevention of risk factors and diseases.
\end{abstract}

Keywords: health promotion; primary health care; health policy.

Trabalho realizado com os profissionais que atuam nas Estratégias Saúde da Família (ESF) - Criciúma (SC), Brasil.

Correspondência: Joni Marcio de Farias. E-mail: jmf@unesc.net

Fonte de financiamento: nenhuma.

Conflito de interesses: nada a declarar.

Recebido em: Ago. 15, 2018. Aprovado em: Ago. 17, 2019

Este é um artigo publicado em acesso aberto (Open Access) sob a licença Creative Commons distribuição e reprodução em qualquer meio, sem restrições desde que o trabalho original seja corretamente citado. 


\section{INTRODUÇÃO}

O movimento de promoção da saúde com mais intensidade a partir de 1974 no Canadá com a divulgação do documento conhecido como Informe de Lalonde ${ }^{1}$, reconhecendo o âmbito multidimensional que determina o processo saúde/doença sistematizado em quatro polos: biologia humana, sistema de organização dos serviços, ambiente e estilo de vida². Em 1978 foi a Conferência Internacional realizada em Alma-Ata, com a necessidade de ações da comunidade mundial em prol da promoção da saúde ${ }^{3}$, ficando mais evidente e concreto a partir da "I Conferência Internacional" realizada em Ottawa em 1986, considerado o termo de referência fundamental no desenvolvimento das propostas de promoção da saúde' ${ }^{1}$, propondo uma agenda internacional com temáticas próprias voltadas ao fortalecimento da promoção a saúde ${ }^{3,4}$.

No Brasil, o Movimento da Reforma Sanitária, iniciado na década de 1970, aponta para a redemocratização da saúde frente às aviltantes desigualdades, a mercantilização da saúde ${ }^{5}$, configurada como ação política centrada no projeto civilizatório de sociedade inclusiva, solidária, tendo a saúde como direito universal de cidadania ${ }^{6}$ e a necessidade de intervenção nos determinantes de saúde ${ }^{7}$. Na década de 1990 a promoção da saúde é contextualizada sobre o pensamento sanitário brasileiro, influenciando estratégias como Cidades Saudáveis e Programa Saúde da Família ${ }^{8}$. Na década de 2000 se intensificam discussões sobre a institucionalização de uma Política Nacional de Promoção da Saúde (PNPS) ${ }^{7}$, instituída pela Portaria MS/GM nº 687, de 30 de março de 2006, revisada no ano de 2014.

A PNPS define promoção da saúde como um conjunto de estratégias e formas de produzir saúde, no âmbito individual/coletivo, que se caracteriza pela articulação e cooperação intra/intersetorial e pela formação da Rede de Atenção à Saúde, com ampla participação e controle social com vistas a promover equidade e melhoria das condições e dos modos de viver, reduzindo vulnerabilidades e riscos à saúde decorrentes dos determinantes sociais, econômicos, políticos, culturais e ambientais 9 .

Apesar de todo o movimento de promoção da saúde realizado nos últimos 25 anos terem provocado avanços em seus significados e práticas, nota-se ainda uma grande prevalência de ações voltadas principalmente à prevenção e recuperação da saúde, ficando à margem as ações de promoção da saúde.

As ações em saúde não seguem necessariamente o que a teoria estipula ou os objetivos de determinados programas, uma vez que sofrem influência da forma de organização do serviço pelas concepções de saúde e de cuidado dos profissionais envolvidos, além das possíveis disputas de interesses e questões políticas que interferem na forma como são realizadas as ações de saúde ${ }^{10}$.

Neste sentido, o estudo apresenta a relação entre o conhecimento dos profissionais sobre a temática de promoção da saúde, estabelecendo uma interface com as práticas do cotidiano do serviço, observando uma contrariedade entre o conhecimento e a pratica, sendo estas muito mais de prevenção a novos agravos e a recuperação da saúde do que de promoção a saúde.

Houve avanços significativos no âmbito das políticas voltadas à promoção da saúde, porém ainda se percebe um distanciamento em termos conceituais e teóricos do significado e da materialização nas práticas de saúde. Diante do exposto, este manuscrito tem o objetivo de identificar o conhecimento sobre promoção da saúde dos profissionais de saúde do município de Criciúma - SC.

\section{MÉTODO}

Trata-se de um estudo observacional descritivo, para avaliar a existência de associação entre o conhecimento teórico e as práticas profissionais de promoção de saúde, sem intervir nas ações realizadas. A pesquisa foi realizada em 28 Estratégias Saúde da Família (ESF), 1 Núcleo de Apoio à Saúde da Família (NASF) e na Secretaria Municipal de Saúde (SMS).

Foram incluídos no estudo trabalhadores da saúde vinculados à Prefeitura Municipal de Criciúma - SC, de ambos os sexos, que responderam a todas as perguntas. Por conseguinte, foram excluídos os participantes que ultrapassaram o prazo solicitado de entrega dos 
questionários. Todos os profissionais receberam o convite e os instrumentos de avaliação em seus locais de trabalho.

Os participantes do estudo foram 123 profissionais de saúde selecionados de forma aleatória, sendo 112 integrantes das Estratégias da Saúde da Família (ESFs) - 20 enfermeiros (E), 13 médicos (M), 12 cirurgiões dentistas (CD) e 67 agentes comunitários de saúde (ACS) -, 3 do NASF - 2 fisioterapeutas (Fi) e 1 farmacêutico (Far) - e 8 coordenadores dos serviços, a saber: saúde mental, saúde bucal, assistência social, NASF, assistência farmacêutica, saúde da mulher e de alimentação e nutrição da SMS.

As falas dos participantes serão identificadas por uma sigla contendo a letra supracitada relativa à categoria do profissional, seguida de um número cardinal e do local de trabalho do participante. Os participantes coordenadores dos serviços serão identificados pela sigla SMS seguida de um número cardinal. A coleta de dados ocorreu entre os meses de agosto a novembro de 2017, por meio de um questionário autoaplicável contendo sete questões abertas sobre o tema promoção da saúde, pautadas na Política Nacional de Promoção da Saúde, elaborado pelos pesquisadores. As avaliações ocorreram durante a jornada de trabalho.

A análise dos dados foi realizada segundo a análise temática de conteúdo, está dividida em três fases ${ }^{11}$, e também por meio de estatística descritiva com utilização de porcentagem ${ }^{12}$. Para o processo organizacional dos dados foi utilizado o software Atlas.ti 8.0, a partir da inserção de documents (documentos), seleção de quotations (trechos de respostas ou citação), criação de codes (códigos) e agrupamento deles e codes group (grupos de códigos) segundo a proximidade temática e por fim foram criadas networks (redes de visualização).

Este estudo foi aprovado pelo Comitê de Ética em Pesquisa da Universidade do Extremo Sul Catarinense - UNESC, parecer n 2.148.413.

\section{RESULTADOS}

Os resultados indicaram um total de 137 quotations e 11 codes, conforme demonstrado na Tabela 1.

Os codes foram estruturados em 4 categorias: modelo preventivo, modelo educativo, modelo positivo de saúde e modelo de articulação, participação e controle social, conforme ilustra a Figura 1.

Tabela 1. Distribuição da quantidade de quotations $(n=137)$, de acordo com os codes $(n=11)$ sobre o entendimento de promoção da saúde dos trabalhadores da saúde do Município de Criciúma - SC, 2017.

\begin{tabular}{|c|c|c|}
\hline \multirow{2}{*}{ Codes } & \multicolumn{2}{|c|}{ Quotations } \\
\hline & $\mathbf{n}$ & $\%$ \\
\hline Prevenção de fatores de risco e doença & 46 & 33,58 \\
\hline Informar e orientar sobre saúde & 25 & 18,25 \\
\hline Melhorar ou manter a saúde e qualidade de vida & 23 & 16,79 \\
\hline Empoderar, conscientizar e ensinar a população/indivíduo & 15 & 10,95 \\
\hline Promover o bem-estar & 11 & 8,03 \\
\hline Processo intersetorial e articulado à população & 6 & 4,38 \\
\hline Cuidados com a saúde & 5 & 3,65 \\
\hline Controlar a saúde & 3 & 2,19 \\
\hline Minimização de danos e agravos à saúde & 1 & 0,73 \\
\hline Estratégia de articulação transversal & 1 & 0,73 \\
\hline Conceito ético/político & 1 & 0,73 \\
\hline Total & 137 & 100,00 \\
\hline
\end{tabular}




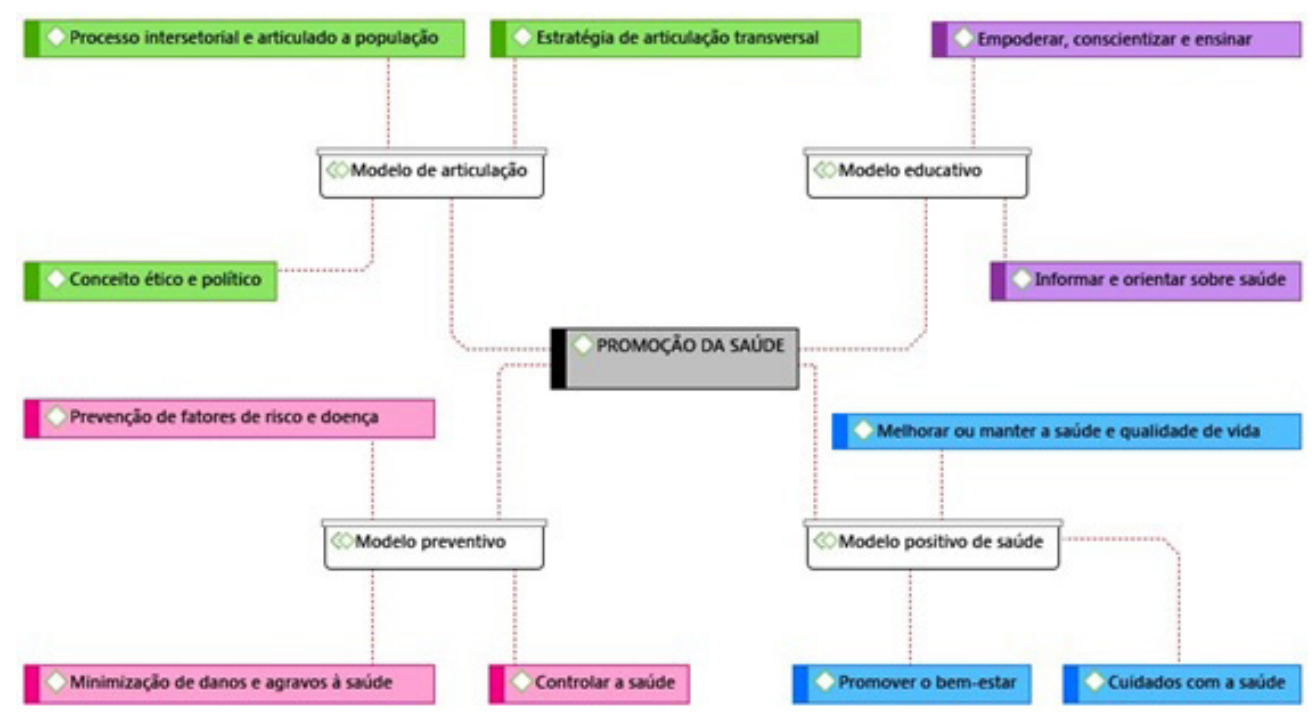

Figura 1. Estrutura de relações sobre o entendimento de promoção da saúde dos profissionais de saúde do município de Criciúma - SC.

\section{Modelo preventivo}

Esta categoria representa $36,6 \%$ das quotations $(n=50)$ e demonstra os seguintes entendimentos sobre promoção da saúde dos profissionais de saúde: prevenção de fatores de risco e doença, controlar a saúde e minimização de danos e agravos à saúde. A presente concepção fica evidenciada nas falas a seguir:

"Ações voltadas para a prevenção de doenças, orientações em grupo, campanhas, consultas de rotina, informativos, controle de esquema vacinal" (M1/ESF);

"Promoção da saúde envolve a prevenção de doenças e agravos, tratamento para quem já foi acometido por alguma doença, e também a manutenção de tudo que interfira na qualidade de vida do indivíduo, desde condições financeiras, moradia, estudo, lazer, etc." (E20/ESF);

"Trabalho voltado a prevenção de agravos relacionados ao processo saúde-doença" (CD12/ESF);

"Prevenção de doenças, tratamento adequado aos doentes, prevenção dos fatores de risco" (ACS2/ESF);

"Um conjunto de estratégia para promover saúde de um aspecto mais preventivo" (Fi1/NASF).

\section{Modelo educativo}

Esta categoria representa $29,2 \%$ das quotations ( $n=40$ ), demonstrando os seguintes entendimentos sobre promoção da saúde dos trabalhadores: informar e orientar sobre saúde e empoderar, conscientizar e ensinar a população/indivíduo. Percebe-se nesta categoria que a prevalência de falas se deu no âmbito de informar e orientar sobre saúde $(n=25)$. As falas a seguir demonstram esses resultados:

"Promoção da saúde é você estar levando algum tipo de informação, conhecimento que você adquiriu para que assim contribuir para a melhora ou manutenção do seu estado de saúde" (ACS22/ESF); 
"Oferecer informações aos pacientes, para que façam consultas de rotina para verificar as suas condições de saúde, não deixar para procurar os serviços de saúde quando já tiver uma doença instalada" (E12/ESF);

"Conjunto de ações e programas que visam orientar a população sobre medidas de cuidado à saúde e controle de patologias" (M10/ESF).

No entanto, falas significativas sobre empoderar e conscientizar o usuário também surgiram nas falas dos profissionais:

"É instrumentalizar o sujeito para tomada de decisão frente suas condições de saúde"(SMS);

"Promoção da saúde é uma espécie de empoderamento que damos aos pacientes, através de ações e repassando o conhecimento de saúde para que eles sejam agentes da sua própria saúde" (ACS20/ESF).

\section{Modelo positivo de saúde}

Esta categoria representa $28,5 \%$ das quotations $(n=39)$ e está estruturada pelos seguintes codes: melhorar ou manter a saúde e qualidade de vida, promover o bem-estar e cuidados com a saúde. As falas descritas a seguir ilustram esta categoria:

"Promover ações que ajudem as pessoas terem, uma qualidade de vida melhor" (ACS11/ESF);

"Um ato ou ação individual ou coletiva para melhorar a qualidade de vida e/ou saúde de uma a várias pessoas" (M9/ESF);

"Promover saúde implica em criar ações para o melhoramento da qualidade de vida e saúde" (E14/ESF).

Falas abordando a promoção do bem-estar e cuidados com a saúde também surgiram:

"Todas as atividades que são realizadas a promover o bem-estar físico, mental e social dos usuários evitando que adoeçam" (E17/ESF);

"Refere-se aos cuidados com a saúde, através de ações de promoção a saúde" (ACS9/ESF).

\section{Modelo de articulação, participação e controle social}

Esta categoria representa $5,8 \%$ das quotations $(n=8)$ e foi elaborada pelo agrupamento dos seguintes codes: processo intersetorial e articulado à população, estratégia de articulação transversal e conceito ético/político. Nesta categoria as falas significativas emergiram com maior prevalência dos gestores de saúde e NASF, que elaboraram definições de promoção da saúde mais complexas e com maior proximidade ao conceito presente na PNPS, como demonstrado a seguir:

"Processo que envolve a população, os trabalhadores da saúde, para que obtenha melhoras na qualidade de vida, no trabalho, nos estudos, nas relações cotidianas, na saúde [...], saber cuidar melhor e também elas mesmas multiplicarem este conhecimento [...]. Precisamos de educação, saneamento, segurança, meio ambiente e muito mais, para nos sentirmos plenos 
com nossa saúde física e mental. Promover, influenciar todos estes meios, podem melhorar a qualidade de vida das pessoas e consequentemente sua saúde" (Far1/NASF);

\begin{abstract}
"Envolvimento da população, profissionais de saúde num processo que todos ou a maioria alcance os índices de melhoras na qualidade de vida [...], prática do cuidar-se melhor e também a multiplicação dos saberes práticos a outras pessoas que os cercam [...]. O estado pleno de saúde física, emocional e mental demonstrara no dia-a-dia [...], necessita de integração saneamento, educação, segurança, meio ambiente" (Far2/NASF);
\end{abstract}

"[...] entendida na sua forma mais ampla de fomentar e incentivar a qualidade de vida e saúde das pessoas por meio de adoção de práticas e hábitos saudáveis em cenários e ambientes que promovam bem-estar e boas condições de saúde [...]. Precisa ser discutida e compreendida para além do setor saúde, através de discussões intersetoriais e formulação de políticas que contemplem os diversos determinantes sociais de saúde, como renda, trabalho, transporte, segurança, lazer" (SMS2).

\title{
DISCUSSÃO
}

Os resultados demonstram o entendimento dos profissionais de saúde sobre promoção da saúde, vinculado a quatro categorias definidas pelas falas mais significativas: modelo preventivo, modelo educativo, modelo positivo de saúde e modelo de articulação, participação e controle social.

Evidencia-se o modelo preventivo com o maior índice de falas, identificando que o entendimento dos trabalhadores da saúde sobre a promoção da saúde está predominantemente relacionado à visão preventivista. Para Czeresnia ${ }^{13}$ a base do discurso preventivo é o conhecimento epidemiológico moderno, seu objetivo é o controle da transmissão de doenças infectocontagiosas e redução do risco de doenças degenerativas ou outros agravos específicos. Deste modo foi possível observar que uma boa parte dos profissionais associa o termo promoção da saúde à prevenção de fatores de risco e doença, sendo até mesmo utilizadas como sinônimos. Essas duas correntes, apesar de ser utilizadas como sinônimos pelos profissionais, são completamente divergentes principalmente no que tange à concepção de saúde, cuja promoção leva em consideração a multidimensionalidade da saúde, enquanto a prevenção a reduz na simples ausência de doenças.

A confusão entre promoção e prevenção advém da grande ênfase em modificações de comportamento individual e do foco quase exclusivo na redução de fatores de risco para determinadas doenças, em centros intitulados de promoção da saúde. Esse modelo fortalece a responsabilidade individual ao sujeito ao mesmo tempo que considera o profissional de saúde como "guru" do modo de viver correto.

A conotação individual fortalecida por este modelo é passível de críticas, uma vez que "[...] não considera a influência do 'social' na determinação, estruturação e padronização das doenças, reduz a saúde, um produto social, a objeto passível de controle do indivíduo" bem como considera "[...] que todas as pessoas vivem nas mesmas condições estruturais e que, assim, todas são igualmente capazes de cuidar de si"14.

Um estudo realizado com profissionais da saúde da Grande Florianópolis identificou que os profissionais compreendem promoção da saúde como prevenção de doenças ${ }^{15}$, corroborando os resultados encontrados nesta pesquisa. Esta dificuldade na compreensão decorre da construção histórica da promoção da saúde em diferentes contextos ${ }^{16}$ e sua aplicação para designar uma face positiva da saúde é consideravelmente recente.

A interface estabelecida entre educação em saúde para promoção está fundamentada PNPS, que compõe o que entendemos pelo modelo educativo. Na análise das falas que abordaram o código, é possível identificar que não há troca de conhecimentos, as informações e orientações são pautadas na linha do profissional como detentor do conhecimento, apenas o transfere ao indivíduo. 
Dagma et al. ${ }^{16}$ descreve que o modelo de educação em saúde é pautado em orientações direcionadas verticalmente ao usuário com uma forte tendência em repassar informações e não reconstruir significados coletivamente. Para Carneiro et al. ${ }^{17}$, em suas observações indicam que as práticas atendem de forma parcial e incipiente os princípios da promoção da saúde. Portanto, precisa-se compreender que educar é um processo, deve ser crítico e problematizador da realidade, construída coletivamente ${ }^{18}$, para informar e orientar sobre saúde, empoderar, conscientizar e ensinar os usuários para o cuidado.

Apesar da prevalência das falas estar relacionada às informações e orientações sobre saúde, também foram levantados códigos a respeito do empoderamento e conscientização do indivíduo, na perspectiva de transmissão de um ator que tem conhecimento e o transfere ao outro desprovido do mesmo. $\mathrm{O}$ empoderamento, como descrito por alguns participantes deste estudo, pode ter um efeito contrário ao proposto pela PNPS, logo, não convergindo na potencialização de ações que visam a materialização nas práticas dos serviços de aspectos relacionados à promoção da saúde.

O empoderamento é uma das estratégias-chave do movimento de promoção da saúde e é considerada uma ferramenta capaz de contribuir para a elaboração de políticas públicas saudáveis e formar sujeitos críticos, autônomos e reflexivos perante a sociedade ${ }^{19}$.

A perspectiva do empoderamento de indivíduos e comunidades vai além da promoção da conscientização, incluindo, também, o fornecimento de informações relevantes ao campo da saúde e habilidades vitais ${ }^{20}$ e ter autonomia para fazer escolhas ${ }^{14}$, o que não foi observado nas falas dos entrevistados.

No modelo positivo de saúde, as falas foram principalmente relacionadas à melhora ou manutenção da saúde e qualidade de vida. Apesar de esses aspectos estarem presentes no discurso da promoção da saúde, eles não definem a promoção da saúde e estão, na verdade, fortemente ligados aos objetivos que se deseja alcançar com as estratégias desse movimento. Ao definir promoção da saúde como ações que melhoram a qualidade de vida, não é deixado claro de que forma se pretenderia chegar a essas condições.

Uma pesquisa realizada por Silva et al. ${ }^{21}$, em Goiânia buscou conhecer os sentidos atribuídos ao conceito de promoção da saúde de 77 gestores de saúde e identificou que 13\% deles entendem promoção da saúde como melhoria da qualidade de vida, sendo encontrado também o fator prevenção e educação em saúde, corroborando este estudo.

O modelo de articulação, participação e controle social foi a categoria que obteve o menor número de quotations. No entanto, levando em consideração o conceito de promoção da saúde presente na PNPS - "Um conjunto de estratégias e formas de produzir saúde, no âmbito individual e coletivo, que se caracteriza pela articulação e cooperação intrassetorial e intersetorial e pela formação da Rede de Atenção à Saúde, buscando se articular com as demais redes de proteção social, com ampla participação e amplo controle social" ${ }^{\prime \prime}$ - é possível analisar uma compreensão mais ampla de promoção da saúde, abordando a intersetorialidade, não restringindo ações de promoção da saúde apenas ao setor saúde. Além de sustentar os determinantes de saúde e criação de políticas públicas e ambientes favoráveis à prática de saúde, aspectos esses considerados até hoje prioridades para o desenvolvimento de ações de promoção da saúde e que vão ao encontro a Carta de Ottawa ${ }^{3}$.

Outro ponto essencial citado foi a intersetorialidade. Este princípio está presente na PNPS como um dos eixos operacionais da promoção da saúde e é fundamental para a elaboração dessas ações, porém sua execução enfrenta muitos desafios. Uma pesquisa realizada por $\mathrm{Silva}^{22}$ em um Centro de Saúde e com equipamentos sociais identificou que existe entre os setores o consenso acerca da importância da intersetorialidade para responder as necessidades da população, no entanto ela ainda é um objetivo a ser alcançado, mas de forma relevante. Foram feitas referências primordiais da comunicação entre os setores para que ela se consagre de forma efetiva.

Não houve predomínio de falas que englobam a amplitude da compreensão sobre promoção da saúde que contemplem preceitos da intersetorialidade, envolvendo a sociedade e a sensibilização dos indivíduos para compreenderem e participarem de forma ativa no seu processo de saúde e na modificação do ambiente que se encontra. 
Os dados evidenciam que empoderamento, intersetorialidade, participação e controle social são elementos primordiais para a construção e desenvolvimento de ações de promoção da saúde, porém foram minimamente citados, sugerindo que os profissionais de saúde possuem uma compreensão de saúde reducionista que se restringe muitas vezes apenas ao setor da saúde.

Os profissionais atuam em grupos definidos como de risco em algumas situações desconsideram o conjunto da população, o ambiente que cerca o usuário bem como tem uma postura frágil no desenvolvimento ações com participação de toda a comunidade para aquisição de hábitos de vida e principalmente as estratégias utilizadas não dialogam com as premissas da promoção da saúde.

O conhecimento e as experiências dos profissionais orientam o modo de fazer saúde em seu território. Neste sentido se faz necessário ampliar as discussões sobre o tema para que ocorra a mudança do "Status Quo", ampliando o conceito de promoção da saúde para além de práticas preventivas reducionistas, fragmentadas, materializadas em sua grande maioria em uma postura de práticas profissionais não condizentes com a PNPS.

\section{REFERÊNCIAS}

1. Czeresnia D, Freitas CM. Promoção da saúde: conceitos, reflexões, tendências. Rio de Janeiro: Fiocruz. 2003.

2. Brasil. Ministério da Saúde. Secretaria de Políticas de Saúde. Projeto promoção da saúde: as cartas da promoção da saúde. Brasília: Ministério da Saúde, 2001.

3. Jorge MR. Declaração de Helsinque [Internet]. França: Associação Médica Mundial; 2013 [citado em 2016 Nov 01]. Disponível em: https://www.wma.net/wp-content/uploads/2016/11/491535001395167888_ DoHBrazilianPortugueseVersionRev.pdf

4. Arouca ASS. Democracia é saúde. In: Brasil. Ministério da Saúde. Brasília, Centro de Documentação Ministério da Saúde. $8^{a}$ Conferência Nacional de Saúde. Brasília: Ministério da Saúde; 1986 [citado em 2017 Out 01] 430p. Disponível em: http://bvsms.saude.gov.br/bvs/publicacoes/0219VIllcns.pdf

5. Souto LRF, Oliveira MHB. Movimento da Reforma Sanitária Brasileira: um projeto civilizatório de globalização alternativa e construção de um pensamento pós-abissal. Saúde Debate. 2016;40(108):20418. http://dx.doi.org/10.1590/0103-1104-20161080017.

6. Catrib AMF, Dias MSA, Frota MA. Promoção da saúde no contexto da estratégia saúde da família. Campinas: Saberes; 2012.

7. Carvalho SR. Saúde Coletiva e promoção da saúde: sujeito e mudança. São Paulo: Editora Hucitec; 2013.

8. Brasil. Ministério da Saúde. Secretaria de Vigilância em Saúde. Secretaria de Atenção à Saúde. Política Nacional de Promoção da Saúde: PNPS: revisão da Portaria MS/GM n 687, de 30 de março de 2006. Brasília: Ministério da Saúde; 2015. p. 7-11.

9. Vasconcelos ACF, Stedefeldt E, Frutuoso MFP. Uma experiência de integração ensino-serviço e a mudança de práticas profissionais: com a palavra, os profissionais de saúde. Interface. 2016;20(56):147-58. http:// dx.doi.org/10.1590/1807-57622015.0395.

10. Soratto J, Friese S, Pires D. Carrying out a computer-aided thematic content analysis with ATLAS.ti. IWMI Working Papers. 2018;18(2). [citado em 2018 Jun 19]. Disponível em: https://www.researchgate.net/ publication/324720405

11. Polit DF, Beck CT. Fundamentos de pesquisa em enfermagem. Avaliação de evidências para a prática da enfermagem. 7. ed. Porto Alegre: Artmed; 2011. (Tradução de Denise Regina de Sales).

12. Czeresnia D, Freitas CM. Promoção da Saúde: conceitos, reflexões e tendências. 2. ed. Rio de Janeiro: Fiocruz; 2009.

13. Oliveira DLA. 'Nova' saúde pública e a promoção da saúde via educação: entre a tradição e a inovação. Rev Latino-am Enfermagem. 2005;13(3):423-31. http://dx.doi.org/10.1590/S0104-11692005000300018.

14. Tesser CD, Garcia AV, Vendruscolo C, Argenta CE. Estratégia saúde da família e análise da realidade social: subsídios para políticas de promoção da saúde e educação permanente. Cien Saude Colet. 2011;16(11):4295-306. http://dx.doi.org/10.1590/S1413-81232011001200002. PMid:22232795.

15. Farinatti PTV, Ferreira MS. Saúde, promoção da saúde e educação física: conceitos, princípios e aplicações. Rio de Janeiro: EdUERJ; 2006. 
16. Costa DW, Pereira BDM, Borges FA, Tavares DMS, Chaves LDP, Goulart BF. Educação em saúde e empoderamento do usuário da estratégia saúde da família. Revista de enfermagem UFPE. 2016;10(1):96102.

17. Carneiro ACLL, Souza V, Godinho LK, Faria ICM, Silva KL, Gazzinelli MF. Educação para a promoção da saúde no contexto da atenção primária. Rev Panam Salud Publica. 2012;31(2):115-20. http://dx.doi.org/10.1590/ S1020-49892012000200004. PMid:22522873.

18. Pelicioni MCF, Pelicioni AF. Educação e promoção da saúde: uma retrospectiva histórica. O Mundo da Saúde. 2007;31(3):320-28.

19. Tones K, Tilford S. Health education: effectiveness, efficiency and equity. 2 nd ed. London (UK): Chapman \& Hall; 1994.

21. Silva RM, Cançado M, Oliveira E, Queiroz MG. Os sentidos do conceito de promoção da saúde na percepção dos gestores da Atenção Primária em Saúde de Goiás, Brasil. CIAIQ. 2017;2:993-1002.

22. Silva $\mathrm{KL}$, Rodrigues AT. Ações intersetoriais para promoção da saúde na Estratégia Saúde da Família: experiências, desafios e possibilidades. Rev Bras Enferm. 2010;63(5):762-9. http://dx.doi.org/10.1590/ S0034-71672010000500011. PMid:21103769. 